\title{
Augmented Reality User Interfaces and Techniques for Outdoor Modelling
}

\author{
Wayne Piekarski and Bruce H. Thomas \\ Wearable Computer Laboratory \\ School of Computer and Information Science \\ University of South Australia \\ Mawson Lakes, SA, 5095, Australia \\ \{wayne, thomas\}@cs.unisa.edu.au
}

\begin{abstract}
This paper presents user interface technology for mobile outdoor augmented reality systems, and a set of new techniques we have named construction at a distance for the capture and creation of $3 \mathrm{D}$ geometry. Using a pair of vision tracked pinch gloves and a specially designed menu system, a user is able to use the physical presence of their body to perform modelling tasks in ways not previously possible.
\end{abstract}

\section{INTRODUCTION}

We have been performing research with mobile outdoor augmented reality (AR) systems and their possible applications. One application that we think is both useful and important is the outdoor modelling of large 3D objects (such as buildings), and the prototyping of plans for new objects that may be constructed in the future. These tasks require complex user interfaces as well as techniques that can model objects that are not directly touchable by the user. This paper demonstrates a novel user interface that uses a pair of vision tracked pinch gloves and a specially designed menu system that can provide a wide set of functionality. Using a set of techniques we have developed known as construction at a distance, an AR system can take advantage of a user's physical presence in the world to capture models of objects that are at a scale larger than the user, and at a distance out of arms reach. Construction at a distance takes previously developed concepts of action at a distance and applies it to the task of creating rather than just editing geometry. The current demonstration in this paper is based on previous work presented in [Piekarski, W. and Thomas, B. 2001] and [Piekarski, W. and Thomas, B. H. 2002].

Operating user interfaces for mobile computers outdoors is inherently difficult due to the large and dynamic nature of the environment. The computing technology must also be portable to allow the user to roam freely in this environment. Restrictions on size, performance, electric power consumption, weight, and magnetic interference limit the number of devices suitable for use outdoors. Although technology improves from year to year, we are designing user interfaces to take advantage of technology available today. Mobile computers can now perform realistic rendering for augmented reality; therefore good user interfaces are now required to support powerful new applications, with particular attention to the limitations of the technology outdoors.

\section{USER INTERFACE}

Mobile outdoor augmented reality does not support the use of many common tracking technologies that are normally used indoors. This also affects the user interfaces that can be used, as many usually require accurate tracking. We have designed a hands free user interface based on pinch gloves that combines command entry and 3D manipulation. Plate 1 shows how the fingers on each hand map to the commands presented on the blue menu strips to the left and right of the display. By pinching the finger against the thumb the appropriate option is selected from a hierarchical menu structure. An important feature is that no tracking is required, and the user can hold their hands wherever is convenient.

The menu is used to represent all the commands available to the user, and many commands do not require any other inputs. We have developed a street furniture application that can place down prefabricated objects anchored in physical space. As the user moves around, these objects appear in the user's view and augment the existing environment. These objects can also be selected and manipulated using menu commands that use a cursor on the user's thumbs - these commands require an object to be selected by the cursor. Figure 1 and Plate 3 show the cursor, and Plates 2 to 4 show these object placement and manipulation examples in operation.

Normally the application is run in an immersive display mode that presents the 3D environment overlaid on the physical world, with 2D displays showing extra information about the current state of the system. We have also developed external VR style views (similar to Koller's orbital view - see Plate 6) with the live video overlay replaced with a sky and ground texture on the HMD. In some techniques this mode is more useful than immersive modes with a limited field of view, as the user has a higher situational awareness of the objects around them. The geometry of many shapes only becomes obvious when viewed from a bird's eye aerial

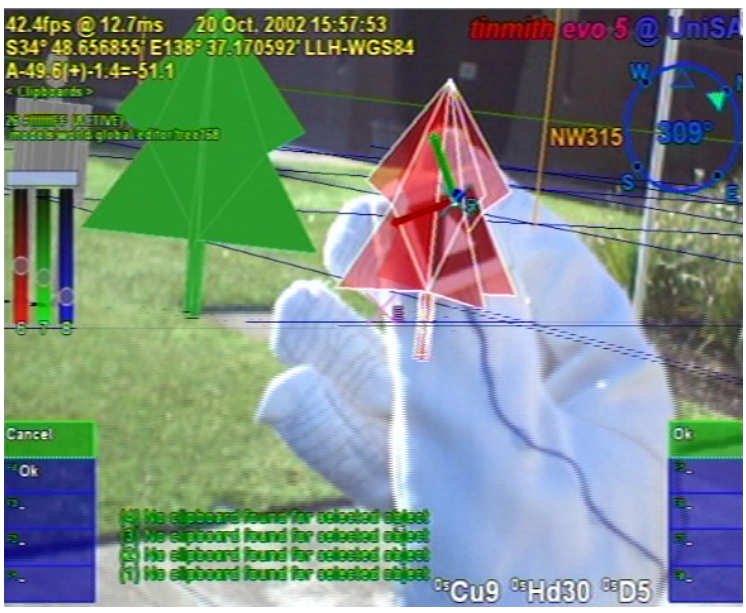

Figure 1 - Immersive augmented reality view, showing a virtual tree being manipulated at a distance using image plane style techniques and optically tracked input gloves 
position instead of at ground level. A caveat of this mode is that the user cannot safely walk around as their view of the physical world is occluded. We found during trials that a VR view with live video background was confusing and hard to understand by the user.

\section{CONSTRUCTION AT A DISTANCE}

We have developed a series of new augmented reality user interaction techniques to support the capture and creation of 3D geometry of large outdoor structures, part of an overall concept we have named construction at a distance. We use information about the user's physical presence, along with hand and head gestures, to capture and create the geometry of objects that are orders of magnitude larger than the user, with no prior information or assistance. Using augmented reality and these new techniques, users can enter geometry and verify its accuracy in real time. Our techniques are based on using simple primitives and operations that are then combined to build up more complex shapes. By iteratively applying modelling operations, the user can refine them based on comparisons made in real time against the physical object. [Brooks, F. P. 1999] describes an iterative refinement process guided by the eye as an efficient way of focusing time onto the areas of the model that require it to capture the right amounts of detail. We define two types of techniques, body relative and $\mathrm{AR}$ working planes that can be combined together in any arbitrary ordering.

\subsection{Body relative techniques}

Using the body and a user's concept of proprioception to improve operations in VR was first explored in [Mine, M. et al. 1997]. We have used this concept to develop a series of techniques that capture geometry of models using just the position of the user's body and the orientation of their head, without any use of the hands. With proprioception the user knows intuitively how to create objects relative to their body. We use constructive solid geometry (CSG carving) to create solids of buildings by placing down simple objects such as planes and boxes, and then combining them to produce more interesting shapes. Infinite carving planes are fired into the world from the user and used to remove portions of infinite solids to

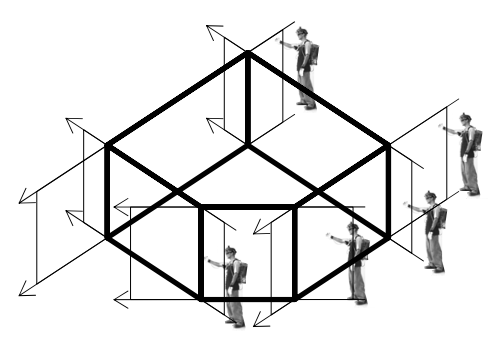

Figure 2 - Infinite carving planes used to carve a convex shape from an infinite solid easily create building outlines (see Figure 2). The bread crumbs technique is used to place points down, and then extrude them up to create arbitrary concave shapes. The texture map capture technique uses the AR overlay camera to map textures onto existing polygons.

\subsection{AR working planes}

Early CAD systems developed a technique known as working planes that can be used to specify 3D points using only 2D input devices and displays. A working plane can be created in 3D space and as the mouse cursor moves across this plane, full $3 \mathrm{D}$ points can be resolved. We have extended this technique to develop $A R$ working planes that can be fired from the body (see previous discussion), and then project points against them from other angles. The laser carving technique projects points against an AR working plane created at the surface of an object, and can orthogonally carve away parts of the object. Laser colouring works similarly but recreates polygons with different colours, and these can be removed to put surface holes into objects. The surface of revolution technique is used to plot points against an AR working plane and then revolve them about a nominated axis to produce round objects such as trees and other similar structures.

\section{IMPLEMENTATION}

The user interface and modelling techniques discussed in this paper have all been implemented in a complete modelling application known as Tinmith-Metro. To implement this application, we use our flexible Tinmith-evo5 software architecture [Piekarski, W. and Thomas, B. H. 2002], which is a complete toolkit for the development of high performance 3D virtual environment applications.

A custom backpack computer was designed to support our research, enabling a single user to carry all the necessary equipment. Our current backpack is known as Tinmith-Endeavour, and is shown in Plate 1 and Plate 2. It is based around a Pentium-III 1.2 $\mathrm{GHz}$ laptop with Nvidia GeForce2, mounted onto a special polycarbonate backplane. An Intersense IS-300 hybrid magnetic and gyroscopic tracker is used for orientation sensing, and a Trimble Ag132 GPS with an accuracy of $50 \mathrm{~cm}$ is used for position sensing. The helmet is based on a Sony Glasstron head mounted display with a FireFly 1394 camera for live video input. The backpack weighs approximately $16 \mathrm{~kg}$ and operates for 2 hours with a $12 \mathrm{~V}$ battery rated at $85 \mathrm{Wh}$. We implemented Tinmith-Endeavour with as many off-the-shelf components as possible, and no effort has been made to miniaturise or lighten the design with custom built components.

Vision tracking of the gloves worn by the user was employed because the system currently already uses a camera to perform live video overlay and texture map capture for augmented reality. The ARToolKit libraries are used to obtain six degree of freedom tracking using small paper fiducial markers attached to the thumbs (see Plate 3 and Plate 6). We use the thumbs for tracking because it is easier to hold the cursor still while pressing it against a finger, compared to finger based markers that would move during presses. Metallic pads on the gloves are used to detect finger presses and control the menu system.

\section{CONCLUSION}

In this paper, we have presented a set of new user interface technologies we have developed to perform complex interactions with mobile outdoor augmented reality systems. We have developed a set of body relative and working plane techniques that we have termed construction at a distance to model real and non-existent objects that are beyond the reach and scale of the user's body.

\section{REFERENCES}

BrooKs, F. P. 1999. What's Real About Virtual Reality? IEEE Computer Graphics and Applications, Vol. 19, No. 6, pp 16-27, Nov 1999.

Mine, M., BRooks, F. P., AND SEQuin, C. H. 1997. Moving Objects In Space: Exploiting Proprioception In Virtual-Environment Interaction. In $A C M$ SIGGRAPH 1997, pp 19-26, Los Angeles, Ca, Aug 1997.

Piekarski, W. And Thomas, B. H. 2001. Tinmith-Metro: New Outdoor Techniques for Creating City Models with an Augmented Reality Wearable Computer. In 5th Int'l Symposium on Wearable Computers, pp 31-38, Zurich, Switzerland, Oct 2001.

PieKarski, W. AND Thomas, B. H. 2002. Tinmith-evo5 - A Software Architecture for Supporting Research Into Outdoor Augmented Reality Environments. Technical Report CIS-02-001, University of South Australia, Adelaide, Jan 2002, http://www.tinmith.net. 


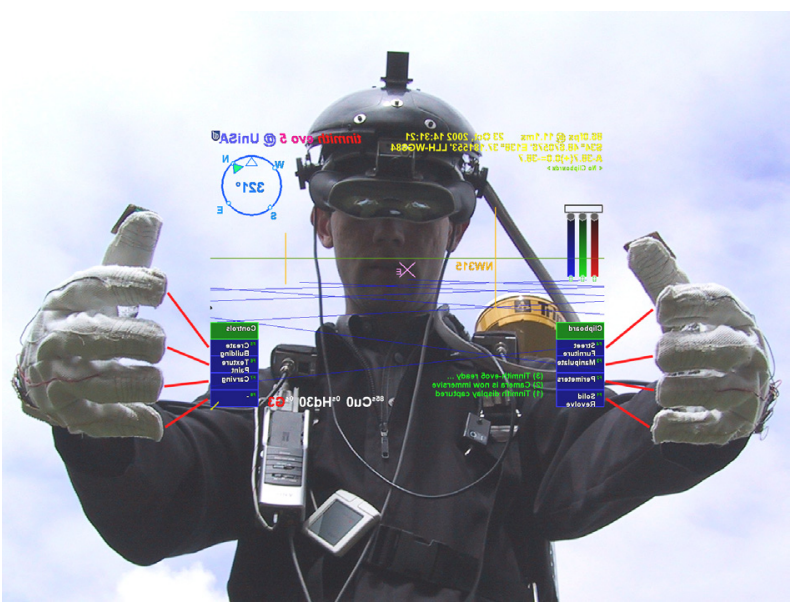

Plate 1 - Each finger maps to a menu option, the user selects one by pressing the appropriate finger against the thumb, and does not rely on the position of the hands when navigating through the menu hierarchy

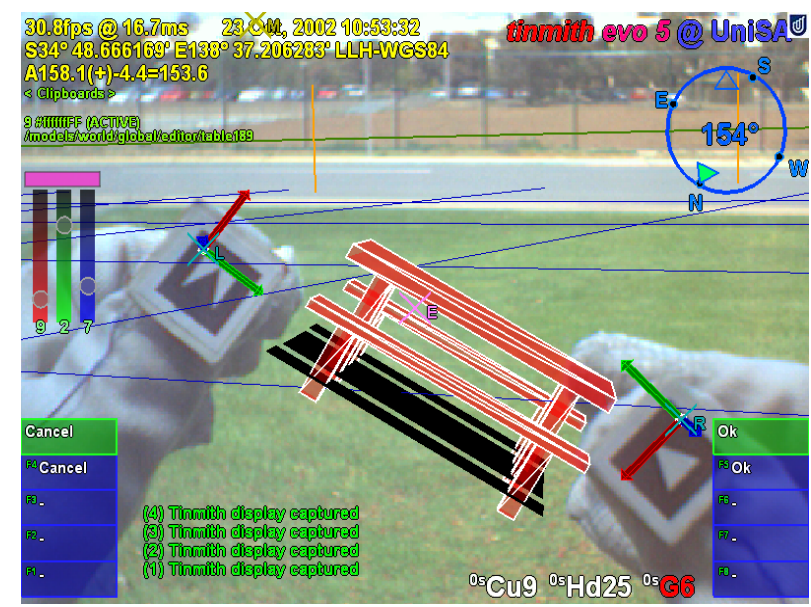

Plate 3 - Immersive AR view of plate 2, showing the virtual table at a distance being rotated with the hands and image plane techniques. The cursors and 2D information overlays are provided for user feedback

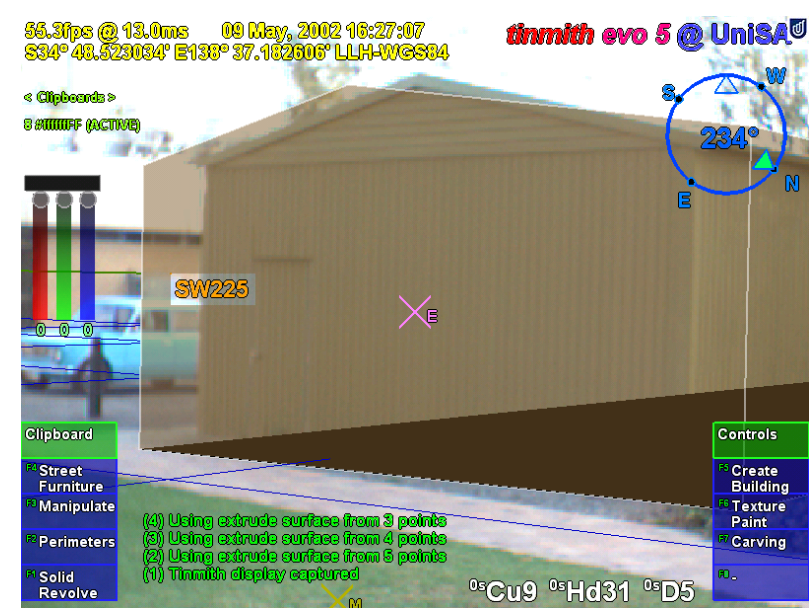

Plate 5 - Transparent 3D model captured using our construction at a distance techniques, overlaid onto the physical world building. This model uses both infinite carving planes and laser carving techniques

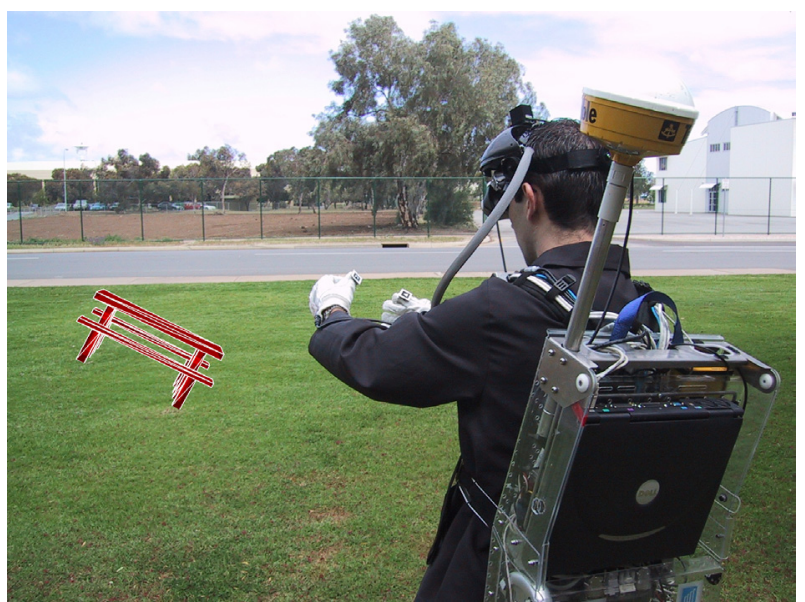

Plate 2 - Example interaction, showing a mobile augmented reality user performing a rotation operation on a virtual table at a distance, wearing the Tinmith-Endeavour backpack to provide real time AR

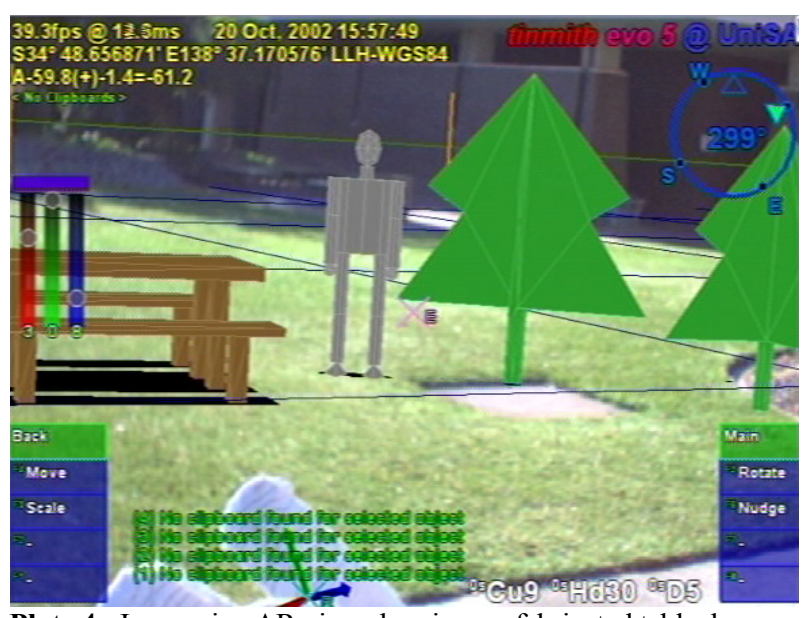

Plate 4 - Immersive AR view showing prefabricated table, human, and tree objects placed down by the user while walking around outside and then registered with the physical world

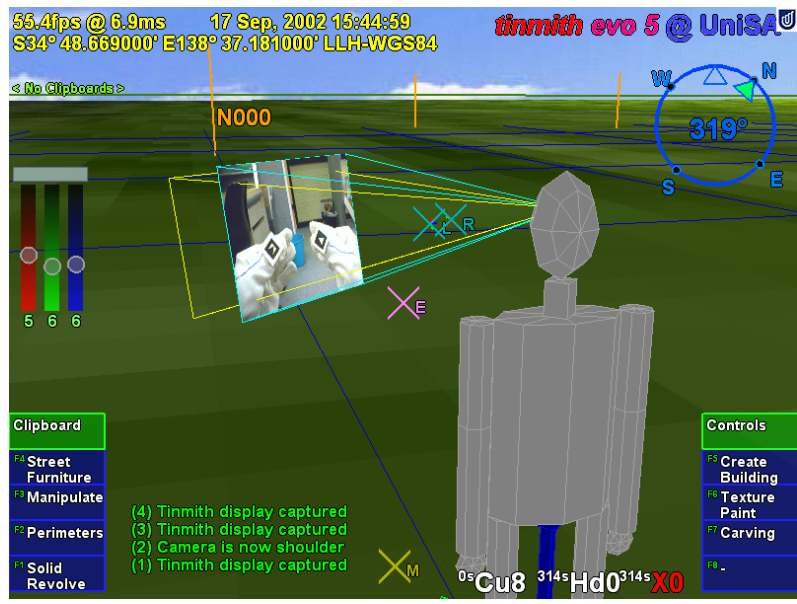

Plate 6 - External VR view, showing the user avatar on a virtual landscape. The view frustum is also shown, with live texture mapped video, and the left $(\mathrm{L})$ and right $(\mathrm{R})$ tracked cursors drawn in $3 \mathrm{D}$ space 